\title{
Special Issue on Selected Papers from the "International Symposium on Remote Sensing 2018"
}

\author{
Hyung-Sup Jung ${ }^{1}{ }^{\oplus}$, Joo-Hyung Ryu ${ }^{2, *}$, Sang-Eun Park ${ }^{3} \oplus$, Hoonyol Lee ${ }^{4}(\mathbb{D}$ and \\ No-Wook Park 5 (D) \\ 1 Department of Geoinformatics, University of Seoul, 163 Seoulsiripdae-ro, Dongdaemun-gu, Seoul 02120, \\ Korea; hsjung@uos.ac.kr \\ 2 Korea Ocean Satellite Center, Korea Institute of Ocean Science \& Technology (KIOST), 385 Haeyang-ro, \\ Yeongdo-gu, Busan Metropolitan City 49111, Korea \\ 3 Department of Geoinformation Engineering, Sejong University, 209 Neungdong-ro, Gwangjin-gu, \\ Seoul 143-747, Korea; separk@sejong.ac.kr \\ 4 Division of Geology and Geophysics, Kangwon National University, Hyoja-dong, Chuncheon, \\ Kangwon-do 24341, Korea; hoonyol@kangwon.ac.kr \\ 5 Department of Geoinformatic Engineering, Inha University, Incheon 22212, Korea; nwpark@inha.ac.kr \\ * Correspondence: jhryu@kiost.ac.kr
}

Received: 14 June 2019; Accepted: 17 June 2019; Published: 18 June 2019

\section{Introduction}

The international symposium on remote sensing 2018 (ISRS 2018) was held in Pyeongchang, Korea, 9-11 May 2018. This symposium has been held in Korea since 1994, and since 2012 it has been officially co-organized by Korean Society of Remote Sensing (KSRS), the Chinese (Taipei) Society of Photogrammetry and Remote Sensing (CSPRS), the Remote Sensing Society of Japan (RSSJ), and the Standing Committee of Environmental Monitoring from Space of East Asia (EMSEA) and has become a special event for the remote sensing field in East Asia. This symposium is a premier symposium that provides all participants with invaluable opportunities to catch up on state-of-the art techniques and the latest developments in remote sensingand provides a platform for sharing new ideas and information with colleagues and young scholars engaged in similar studies, research, or activities.

During the 2018 symposium, there were 39 sessions with a total of 258 papers (156 orals, 102 posters). Among these papers, 123 oral papers were assigned to 31 general sessions on various aspects of remote sensing, from systems including microwave and optical sensors, to land, ocean, atmosphere, and cryosphere applications. In addition, 33 oral papers were presented in the workshop on "Advanced Technology for Satellite-based Water Resources Management" by the K-water, as well as six Special Sessions, which included one session on "Advanced Environmental Management using Environmental Spatial Information and Remote Sensing" by the Ministry of Environment, four sessions on "Geostationary Ocean Color Mission" organized by the Korea Institute of Ocean Science and Technology (KIOST), and, finally, one session on "KOMPSAT Applications" by the Korea Aerospace and Research Institute (KARI).

This Special Issue on Selected Papers from the "International Symposium on Remote Sensing 2018" in Remote Sensing was planned in conjunction with ISRS 2018 and includes peer-reviewed feature papers presented at ISRS 2018. The ISRS 2018 conference papers were fleshed out with more detailed presentations of the research and also additional data sets and comparisons in enhanced experimental sections. Finally, eighteen papers that reflect the topics of interest for this special issue have been accepted for publication in this special issue of Remote Sensing. 


\section{Remote Sensing Technology and Its Applications}

In this special issue, we have selected eighteen papers that reflect topics of interest in the remote sensing field. The published papers are introduced as follows:

Kadavi et al. [1], in their paper entitled "Application of Ensemble-Based Machine Learning Models to Landslide Susceptibility Mapping", applied four ensemble-based machine learning models, including the AdaBoost, LogitBoost, Multiclass Classifier, and Bagging models, to landslide susceptibility mapping and evaluated the performance of the models using statistical evaluation measures, the receiver operating characteristic (ROC) curve, and the area under the curve (AUC).

Lee and Kim [2], in their paper entitled "A Performance Evaluation of a Geo-Spatial Image Processing Service Based on Open Source PaaS Cloud Computing Using Cloud Foundry on OpenStack", built a Platform-as-a-Service (PaaS) based service, which is linked with the Open Geospatial Consortium (OGC) Web Processing Service (WPS) 2.0 and tested on the basis of the performance results of an edge extraction algorithm against Infrastructure-as-a-Service (IaaS) based service with the same function. In conclusion, they revealed that the PaaS-based geo-spatial service had greater performance.

Park et al. [3], in their paper entitled "Landslide Susceptibility Mapping and Comparison Using Decision Tree Models: A Case Study of Jumunjin Area, Korea", created landslide susceptibility maps using three decision tree models- the Chi-square Automatic Interaction Detection (CHAID), exhaustive CHAID, and Quick, Unbiased and Efficient Statistical Tree (QUEST) models-in Jumunjin-eup, Gangneung-si, Korea and evaluated the performance of the three models by using the AUC method.

Lee et al. [4], in their paper entitled "Vegetation Height Estimate in Rice Fields Using Single Polarization TanDEM-X Science Phase Data", proposed an efficient method to retrieve rice paddy height by employing single polarization (single-pol) synthetic aperture radar interferometry (InSAR) using model-based height inversion without an existing digital elevation model (DEM). They showed that the correlation coefficient of the proposed method was about 0.78 and the root mean square error (RMSE) was about $0.10 \mathrm{~m}$.

Ghareshelou et al. [5] in their paper entitled "A Simple Method for the Parameterization of Surface Roughness from Microwave Remote Sensing" measured and evaluated the surface roughness parameter for the SAR backscattering model in an agricultural field, using images acquired from a hand-held camera in order to support the SAR soil moisture retrieval.

Mak et al. [6], in their paper entitled "Improved Satellite Retrieval of Tropospheric $\mathrm{NO}_{2}$ Column Density via Updating of Air Mass Factor (AMF): Case Study of Southern China", combined China land-use data with meteorological and chemical information and then retrieved the tropospheric $\mathrm{NO}_{2}$ vertical column density (VCD) for the different seasons of 2015 over Southern China. In this paper, the meteorological and chemical information was derived from the Weather Research and Forecasting (WRF) and Community Multiscale Air Quality (CMAQ) models using the Berkeley High Resolution (BEHR) approach.

Hwang and Jung [7], in their paper entitled "Automatic Ship Detection Using the Artificial Neural Network and Support Vector Machine from X-Band SAR Satellite Images", suggested an improved ship detection method based on artificial neural network (ANN) and support vector machine (SVM) techniques from X-band COSMO-SkyMed, KOMPSAT-5, and TerraSAR-X SAR images. For this study, the authors generated two optimal input images-intensity and texture difference maps-and then used the maps for the input neurons of the ANN and SVM approaches to improve ship detection performance.

Jung et al. [8], in their paper entitled "Comparison of Pre-Event VHR Optical Data and Post-Event PolSAR Data to Investigate Damage Caused by the 2011 Japan Tsunami in Built-Up Areas", suggested an efficient method to assess disaster-induced damage in built-up areas using the difference between pre-disaster optical data and post-disaster PolSAR data. The authors developed the proposed method based on a simulation approach to effectively find the difference between pre-disaster optical data and post-disaster PolSAR data. 
Song et al. [9], in their paper entitled "Change Detection in Hyperspectral Images Using Recurrent 3D Fully Convolutional Networks", proposed a sample generation method and a novel change detection network, which is named a recurrent three-dimensional (3D) fully convolutional network (Re3FCN). The Re3FCN model is a fully convolutional network (FCN), including 3D convolutional layers and a convolutional long short-term memory (LSTM).

Hong et al. [10], in their paper entitled "Using TanDEM-X Pursuit Monostatic Observations with a Large Perpendicular Baseline to Extract Glacial Topography", performed a feasibility test to construct high-resolution and high-precision topographic elevation models from TanDEM-X pursuit monostatic observations during the TanDEM-X Science Phase. For this study, the authors evaluated the measurement performance from more detailed topographic height variations along the moving surfaces of low slopes like glaciers.

Hsu et al. [11], in their paper entitled "Developing Land-Use Regression Models to Estimate $\mathrm{PM}_{2.5}$-Bound Compound Concentrations", applied land-use regression (LUR) models to a $\mathrm{PM}_{2.5}$-bound compound concentration based on the measurements of a six-year period using remote sensing (RS) and geographical information system (GIS) techniques. Their results showed that the RS and GIS technologies can be used for the exposure assessment of $\mathrm{PM}_{2.5}$-bound compounds.

Kim et al. [12], in their paper entitled "Satellite-Based Prediction of Arctic Sea Ice Concentration Using a Deep Neural Network with Multi-Model Ensemble", suggested a new approach for the near-future prediction of Arctic sea ice concentration based on the deep neural network (DNN) model implemented with a multi-model ensemble.

Kim et al. [13], in their paper entitled "Digital Surface Model Interpolation Based on 3D Mesh Models", proposed a digital surface model (DSM) interpolation method based on a 3D mesh model. The proposed method has the advantage that it is more robust against outliers and large holes, even though there is no a priori knowledge of surface types.

Shin et al. [14], in their paper entitled "Synergistic Effect of Multi-Sensor Data on the Detection of Margalefidinium polykrikoides in the South Sea of Korea", performed a feasibility test to detect Margalefidinium polykrikoides blooms by identifying a possible synergistic effect of multi-sensor data. Their results showed that the detection performance of Margalefidinium polykrikoides blooms increased when multi-sensor data were used.

Yoon and Kim [15], in their paper entitled "Development of Stereo Visual Odometry Based on Photogrammetric Feature Optimization", developed an efficient photogrammetric feature optimization method that can be applied to stereo visual odometry (SVO). The developed method has the advantage that it can perform real-time outlier removal through a photogrammetric analysis.

Kim et al. [16], in their paper entitled "Multi-Temporal Analysis of Forest Fire Probability Using Socio-Economic and Environmental Variables", considered multiple socio-economic factors to predict and analyze the spatial distribution of forest fire probability in South Korea. For this study, the authors (1) predicted and analyzed the spatial distribution using both Maxent and Random Forest models based on a multi-temporal data analysis, (2) compared between results from the two models, and (3) determined the relationship between forest fire probability and socio-economic factors.

Shiu and Chuang [17], in their paper entitled "Yield Estimation of Paddy Rice Based on Satellite Imagery: Comparison of Global and Local Regression Models", proposed an efficient approach to estimate paddy rice yield using (1) the global regression models including the ordinary least squares (OLS) and support vector regression (SVR) and (2) the local model of geographically weighted regression (GWR).

Kim et al. [18], in their paper entitled "Generation of a Large-Scale Surface Sediment Classification Map Using Unmanned Aerial Vehicle (UAV) Data: A Case Study at the Hwang-do Tidal Flat, Korea", classified a large-scale surface sediment from high spatial resolution UAV images. The achieved overall accuracy was about $72.8 \%$ with a kappa coefficient of 0.62 . Their result showed that UAV images can be used to classify a large-scale surface sediment. 
Acknowledgments: We are very thankful for the contributions of the professional authors and reviewers and the excellent assistant of the editorial team of Remote Sensing.

\section{References}

1. Kadavi, P.R.; Lee, C.-W.; Lee, S. Application of Ensemble-Based Machine Learning Models to Landslide Susceptibility Mapping. Remote Sens. 2018, 10, 1252. [CrossRef]

2. Lee, K.; Kim, K. A Performance Evaluation of a Geo-Spatial Image Processing Service Based on Open Source PaaS Cloud Computing Using Cloud Foundry on OpenStack. Remote Sens. 2018, 10, 1274. [CrossRef]

3. Park, S.-J.; Lee, C.-W.; Lee, S.; Lee, M.-J. Landslide Susceptibility Mapping and Comparison Using Decision Tree Models: A Case Study of Jumunjin Area, Korea. Remote Sens. 2018, 10, 1545. [CrossRef]

4. Lee, S.-K.; Yoon, S.Y.; Won, J.-S. Vegetation Height Estimate in Rice Fields Using Single Polarization TanDEM-X Science Phase Data. Remote Sens. 2018, 10, 1702. [CrossRef]

5. Gharechelou, S.; Tateishi, R.; Alan Johnson, B. A Simple Method for the Parameterization of Surface Roughness from Microwave Remote Sensing. Remote Sens. 2018, 10, 1711. [CrossRef]

6. Mak, H.W.L.; Laughner, J.L.; Fung, J.C.H.; Zhu, Q.; Cohen, R.C. Improved Satellite Retrieval of Tropospheric NO2 Column Density via Updating of Air Mass Factor (AMF): Case Study of Southern China. Remote Sens. 2018, 10, 1789. [CrossRef]

7. Hwang, J.-I.; Jung, H.-S. Automatic Ship Detection Using the Artificial Neural Network and Support Vector Machine from X-Band Sar Satellite Images. Remote Sens. 2018, 10, 1799. [CrossRef]

8. Jung, M.; Yeom, J.; Kim, Y. Comparison of Pre-Event VHR Optical Data and Post-Event PolSAR Data to Investigate Damage Caused by the 2011 Japan Tsunami in Built-Up Areas. Remote Sens. 2018, 10, 1804. [CrossRef]

9. Song, A.; Choi, J.; Han, Y.; Kim, Y. Change Detection in Hyperspectral Images Using Recurrent 3D Fully Convolutional Networks. Remote Sens. 2018, 10, 1827. [CrossRef]

10. Hong, S.-H.; Wdowinski, S.; Amelung, F.; Kim, H.-C.; Won, J.-S.; Kim, S.-W. Using TanDEM-X Pursuit Monostatic Observations with a Large Perpendicular Baseline to Extract Glacial Topography. Remote Sens. 2018, 10, 1851. [CrossRef]

11. Hsu, C.-Y.; Wu, C.-D.; Hsiao, Y.-P.; Chen, Y.-C.; Chen, M.-J.; Lung, S.-C.C. Developing Land-Use Regression Models to Estimate PM2.5-Bound Compound Concentrations. Remote Sens. 2018, 10, 1971. [CrossRef]

12. Kim, J.; Kim, K.; Cho, J.; Kang, Y.Q.; Yoon, H.-J.; Lee, Y.-W. Satellite-Based Prediction of Arctic Sea Ice Concentration Using a Deep Neural Network with Multi-Model Ensemble. Remote Sens. 2019, 11, 19. [CrossRef]

13. Kim, S.; Rhee, S.; Kim, T. Digital Surface Model Interpolation Based on 3D Mesh Models. Remote Sens. 2019, 11, 24. [CrossRef]

14. Shin, J.; Kim, K.; Son, Y.B.; Ryu, J.-H. Synergistic Effect of Multi-Sensor Data on the Detection of Margalefidinium polykrikoides in the South Sea of Korea. Remote Sens. 2019, 11, 36. [CrossRef]

15. Yoon, S.-J.; Kim, T. Development of Stereo Visual Odometry Based on Photogrammetric Feature Optimization. Remote Sens. 2019, 11, 67. [CrossRef]

16. Kim, S.J.; Lim, C.-H.; Kim, G.S.; Lee, J.; Geiger, T.; Rahmati, O.; Son, Y.; Lee, W.-K. Multi-Temporal Analysis of Forest Fire Probability Using Socio-Economic and Environmental Variables. Remote Sens. 2019, 11, 86. [CrossRef]

17. Shiu, Y.-S.; Chuang, Y.-C. Yield Estimation of Paddy Rice Based on Satellite Imagery: Comparison of Global and Local Regression Models. Remote Sens. 2019, 11, 111. [CrossRef]

18. Kim, K.-L.; Kim, B.-J.; Lee, Y.-K.; Ryu, J.-H. Generation of a Large-Scale Surface Sediment Classification Map Using Unmanned Aerial Vehicle (UAV) Data: A Case Study at the Hwang-do Tidal Flat, Korea. Remote Sens. 2019, 11, 229. [CrossRef] 